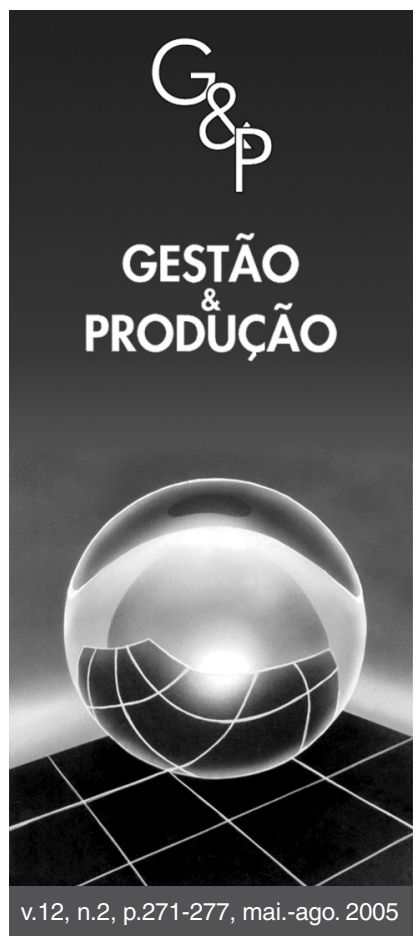

\title{
O USO DA ESTATÍSTICA DE QUI-QUADRADO NO CONTROLE DE PROCESSOS
}

\author{
Antônio Fernando Branco Costa \\ FEG/UNESP, Avenida Ariberto Pereira da Cunha, 333, CEP 12516-410, \\ Bairro do Pedregulho, Guaratinguetá, SP. \\ e-mail: fbranco@feg.unesp.br \\ Maysa Sacramento de Magalhães \\ ENCE/IBGE, Rua André Cavalcanti, 106, CEP 20231-050, \\ Bairro Santa Teresa, Rio de Janeiro, RJ. \\ e-mail: maysa@ibge.gov.br
}

Resumo

Dois gráficos de controle são, usualmente, utilizados no monitoramento da média e da variância de um processo. Em geral, utiliza-se o gráfico de $\bar{X}$ para a detecção de alterações da média, e o gráfico de $R$ para a sinalização de aumentos da variabilidade. Neste artigo, propõe-se o uso de uma única estatística e, portanto, de um único gráfico, como alternativa à prática comum do monitoramento de processos por meio de dois gráficos de controle. O gráfico proposto, baseado na estatística de Qui-quadrado não-central, tem se mostrado mais eficiente que os gráficos de $\bar{X}$ e $R$. Além disso, se as decisões sobre as condições dos parâmetros do processo são baseadas no histórico das observações e não apenas na última observação, então o uso da estatística de Qui-quadrado não-central é indicado para a detecção de pequenas perturbações. Neste estudo, são também apresentados os gráficos de controle da média móvel ponderada exponencialmente (EWMA) baseados na estatística Qui-quadrado não-central.

Palavras-chave: gráfico de controle de Qui-quadrado, gráficos conjuntos de $\bar{X}$ e $R$, número médio de amostras até o sinal.

\section{Introdução}

Nos últimos anos, uma grande atenção tem sido devotada ao estudo das propriedades conjuntas dos gráficos de controle destinados ao monitoramento da média e da variância do processo. Por exemplo, Costa (1993), Costa e Rahim (2000, 2001) e Rahim e Costa (2000) desenvolveram modelos econômicos para os gráficos conjuntos de $\bar{X}$ e R. Gan (1995) estudou as propriedades conjuntas de dois gráficos de controle EWMA. Albin et al. (1997) estudou os gráficos conjuntos, $X$ e $E W M A$, para observações individuais. Chen et al. (2001) combinou dois gráficos de controle de EWMA em um único e mostrou que o novo gráfico de $E W M A$ é eficiente na detecção de aumentos e decréscimos da média e/ou da variância do processo. Costa (1998 e 1999) estudou os gráficos conjuntos de $\bar{X}$ e $R$ com parâmetros variáveis, e Costa e Rahim (2004) estudaram os gráficos conjuntos de $\bar{X}$ e $\mathrm{R}$ com amos- tragens em dois estágios. Reynolds e Stoumbos (2001) investigaram três gráficos conjuntos destinados ao monitoramento da média e da variância de uma variável normal, quando uma observação individual é tomada a cada instante de amostragem. Eles consideraram o gráfico das observações individuais $X$ em uso conjunto com o gráfico da amplitude móvel $(M R)$, o gráfico das observações individuais $X$ em uso conjunto com um gráfico de $E W M A$, e dois gráficos de EWMA em uso conjunto; sendo que um deles utiliza as observações diretamente, enquanto o outro utiliza a raiz quadrada dos desvios das observações em relação a um valor especificado (valor alvo). A principal conclusão que pode ser tirada de todos esses estudos é que nenhum dos gráficos conjuntos é confiável na identificação do tipo de causa especial. Em outras palavras, sempre que uma causa especial é detectada, 
nunca se sabe se tal causa especial é aquela que apenas altera a média, ou apenas aumenta a variabilidade, ou, no pior caso, altera a média aumentando a variabilidade. Por exemplo, quando os gráficos conjuntos de $\bar{X}$ e $R$ estão em uso, e o gráfico de $\bar{X}$ sinaliza a presença de uma causa especial, então se deve investigar qual parâmetro do processo foi afetado pela causa especial, pois o gráfico de $\bar{X}$ é sensível não só a mudanças da média, como também a aumentos da variabilidade.

Na prática, a velocidade com a qual os gráficos de controle detectam mudanças no processo parece ser mais importante do que a habilidade destes em identificar o tipo de mudança. Deste modo, faz sentido considerar um único gráfico de controle, baseado em uma única estatística, para o monitoramento simultâneo da média e da variância do processo. Esse é o caso do gráfico de controle proposto por Domangue e Patch (1991), que é excelente na detecção de mudanças da média e/ou da variância. Shamma e Amin (1993) consideraram o gráfico proposto por Domangue e Patch nas condições em que o intervalo de tempo entre a retirada de amostras é variável.

Neste trabalho, propõe-se o uso de uma única estatística, portanto de um único gráfico de controle, para o monitoramento simultâneo da média e da variância do processo. Trata-se de um gráfico de Shewhart, pois as decisões, sobre as condições dos parâmetros do processo, são sempre tomadas com base apenas nas observações da última amostra. Em termos de comparação com os gráficos de $\bar{X}$ e $R$, o gráfico proposto é mais simples de se utilizar, e mais ágil na detecção de causas especiais que alteram a média e/ou aumentam a variabilidade do processo. Propõe-se também o gráfico individual de EWMA, baseado na estatística de Qui-quadrado não-central. Trata-se de uma ferramenta poderosa na detecção de pequenas mudanças nos parâmetros do processo.

A estatística $W$, a ser utilizada no monitoramento do processo, e o procedimento para a obtenção das propriedades do gráfico proposto compõem a próxima seção. A seção 3 estuda as propriedades do gráfico de controle EWMA baseado na estatística $W$, e a seção 4 apresenta um exemplo de aplicação do gráfico de EWMA.

\section{Propriedades do gráfico de controle de $\chi^{2}(\lambda)$}

Assume-se, neste trabalho, que gráficos de controle são utilizados no monitoramento de processos cuja característica de qualidade de interesse $X$ tem distribuição normal com média $\mu$ e desvio padrão $\sigma$. Quando o processo está sob controle, ou seja, isento de causas especiais, $\mu=\mu_{0}$ e $\sigma=\sigma_{0}$. A causa especial desloca a média $\mu$ de seu valor alvo $\mu_{0}$ para $\mu_{1}=\mu_{0} \pm \delta \sigma_{0}$, em que $\delta \neq 0$, e/ou altera o desvio padrão $\sigma$ de $\sigma_{0}$ para $\sigma_{1}=\gamma \sigma_{0}$, em que $\gamma>1$.
Seja $x_{i j}, i=1,2,3, \ldots, \mathrm{e} j=1,2, \ldots, n$ as medidas da variável $X$, organizadas em subgrupos de tamanho $n$, sendo $i$ o número do subgrupo, e seja

$$
\xi_{i}=d \text { se } \sum_{j=1}^{n} x_{i j} \geq n \mu_{0} ; \text { caso contrário } \xi_{i}=-d,
$$

em que $d$ é uma constante positiva.

Os pontos amostrais $W_{i}$, do gráfico de controle de Quiquadrado não-central (gráfico de $\chi^{2}(\lambda)$ ), são dados por

$$
W_{i}=\sum_{j=1}^{n}\left(x_{i j}-\mu_{0}+\xi_{i} \sigma_{0}\right)^{2}, i=1,2, \ldots
$$

Condicionado a um valor de $\xi_{i}, W_{i} / \sigma_{0}^{2}$, tem, para $\mu=\mu_{0}$ e para $\sigma=\sigma_{0}$, distribuição de Qui-quadrado não-central com $n$ graus de liberdade e parâmetro de não centralidade $\lambda_{0}=n d^{2}$, e para $\mu=\mu_{1}$ e $\sigma=\sigma_{1}, W_{i} / \sigma_{1}^{2}$ tem distribuição de Qui-quadrado não-central com $n$ graus de liberdade e parâmetro de não centralidade $\lambda_{1}=n\left(\delta+\xi_{i}\right)^{2} / \gamma^{2}$, ou seja, $W_{i} / \sigma_{0}^{2} \sim \chi_{n}^{2}\left(\lambda_{0}\right)$ e $W_{i} / \sigma_{1}^{2} \sim \chi_{n}^{2}\left(\lambda_{1}\right)$. O gráfico proposto sinaliza uma condição de falta de controle, sempre que $W_{i}>k_{\mathrm{Chi}} \sigma_{0}^{2}$, em que $k_{\mathrm{Chi}}$ é o fator de abertura do limite superior de controle do gráfico. Como $\xi_{i}$ é uma variável aleatória, que depende do valor de $\bar{X}_{i}$, obtém-se o risco $\alpha$ e o poder de detecção do gráfico proposto por meio da seguinte expressão:

$P_{S}=\int_{-\infty}^{\infty} \operatorname{Pr}\left\{\chi_{n-1}^{2}>\left[k_{C h i} / a-(z+b \sqrt{n})^{2}\right]\right\} \phi(z) d z$

em que $\phi(z)$ é a função densidade de probabilidade da distribuição normal padrão. Quando o processo está sob controle $a=1, b=d$, se $z>0$, caso contrário $b=-d$, e o risco de alarme falso $\alpha=P_{\mathrm{S}}$. Quando o processo está fora de controle $a=\gamma^{2}, b=(\delta+d) / \gamma$, se $z>-\delta \sqrt{n} / \gamma$, caso contrário $b=(\delta-d) / \gamma$, e o poder do gráfico de controle $P d=P_{S}$.

A capacidade de um gráfico de controle detectar mudanças no processo pode ser medida pelo número médio de amostras até o sinal, NMA, (Costa et al., 2004). O NMA para o gráfico proposto é facilmente determinado, pois, neste caso, o número de amostras até o sinal é uma variável aleatória com distribuição geométrica de parâmetro $P_{S}$, isto é, $N M A=1 / P_{S}$. Durante o período em controle, $N M A=N M A_{0}=1 / \alpha$.

A Tabela 1 fornece o NMA dos gráficos de $\chi^{2}(\lambda)$ e de $\bar{X}$ e $R$ para o seguinte conjunto de valores: $N M A_{0}=433,0$; $n=4 ; 5, d=0 ; 0,5$ e $1, \delta=0 ; 0,5 ; 0,75 ; 1,00 ; 1,25$ e 1,50 , e $\gamma=1,0 ; 1,3 ; 1,5$ e 2,0. Com $d=1$ o gráfico de $\chi^{2}(\lambda)$ é sempre mais ágil que os gráficos de $\bar{X}$ e $R$, na detecção de causas especiais que alteram a média do processo e/ou aumentam a variabilidade. Os valores do NMA foram obtidos com o auxílio da estação de trabalho FORTRAN 4.0 da Microsoft.

Para um dado valor de $n$ e de $d$, o NMA do gráfico proposto e dos gráficos conjuntos de $\bar{X}$ e $R$ diminuem 
Tabela 1. Valores do $N M A$ para os gráficos de $\bar{X}$ e $\boldsymbol{R}$ e para o gráfico de $\chi^{2}(\lambda)$.

\begin{tabular}{|c|c|c|c|c|c|c|c|c|c|}
\hline \multirow[b]{4}{*}{$\delta$} & \multicolumn{6}{|c|}{$n=4$} & \multicolumn{3}{|c|}{$n=5$} \\
\hline & & $d=$ & $\mathbf{0 , 0}$ & 0,5 & 1,0 & & $\mathbf{0 , 0}$ & 0,5 & 1,0 \\
\hline & & $\boldsymbol{k}_{C h i}=$ & 16,602 & 21,836 & 30,084 & & 18,572 & 24,352 & 33,871 \\
\hline & $\gamma$ & $\bar{X}-\boldsymbol{R}^{*}$ & \multicolumn{7}{|c|}{$\bar{X}-\boldsymbol{R}^{* *}$} \\
\hline \multirow[t]{4}{*}{0,00} & 1,0 & 433,0 & 433,0 & 433,0 & 433,0 & 433,0 & 433,0 & 433,0 & 433,0 \\
\hline & 1,3 & 29,2 & 23,0 & 25,1 & 29,2 & 25,9 & 19,4 & 21,5 & 25,8 \\
\hline & 1,5 & 10,9 & 8,5 & 9,3 & 10,9 & 9,5 & 7,0 & 7,7 & 9,3 \\
\hline & 2,0 & 3,1 & 2,6 & 2,7 & 3,1 & 2,6 & 2,0 & 2,3 & 2,6 \\
\hline \multirow[t]{4}{*}{0,50} & 1,0 & 74,8 & 115,2 & 70,0 & 58,4 & 56,6 & 104,3 & 56,8 & 45,6 \\
\hline & 1,3 & 15,8 & 13,9 & 12,5 & 12,7 & 13,2 & 11,5 & 10,0 & 10,3 \\
\hline & 1,5 & 7,8 & 6,4 & 6,3 & 6,8 & 6,6 & 5,3 & 5,2 & 5,6 \\
\hline & 2,0 & 2,8 & 2,4 & 2,5 & 2,7 & 2,4 & 2,0 & 2,1 & 2,3 \\
\hline \multirow[t]{4}{*}{0,75} & 1,0 & 24,3 & 42,3 & 24,2 & 19,9 & 16,9 & 35,5 & 19,4 & 14,6 \\
\hline & 1,3 & 9,2 & 8,8 & 7,3 & 7,1 & 7,3 & 7,2 & 5,8 & 5,6 \\
\hline & 1,5 & 5,6 & 4,9 & 4,5 & 4,6 & 4,7 & 4,0 & 3,7 & 3,8 \\
\hline & 2,0 & 2,5 & 2,2 & 2,2 & 2,4 & 2,2 & 1,9 & 1,9 & 2,0 \\
\hline \multirow[t]{4}{*}{1,00} & 1,0 & 9,4 & 16,6 & 9,4 & 8,2 & 6,4 & 13,2 & 7,2 & 5,9 \\
\hline & 1,3 & 5,4 & 5,5 & 4,5 & 4,3 & 4,2 & 4,5 & 3,5 & 3,3 \\
\hline & 1,5 & 4,0 & 3,6 & 3,2 & 3,2 & 3,3 & 3,0 & 2,6 & 2,6 \\
\hline & 2,0 & 2,2 & 2,0 & 2,0 & 2,1 & 1,9 & 1,7 & 1,7 & 1,8 \\
\hline \multirow[t]{4}{*}{1,25} & 1,0 & 4,4 & 7,4 & 4,5 & 4,0 & 3,1 & 5,7 & 3,5 & 3,0 \\
\hline & 1,3 & 3,4 & 3,6 & 3,0 & 2,8 & 2,6 & 2,9 & 2,4 & 2,2 \\
\hline & 1,5 & 2,8 & 2,7 & 2,4 & 2,4 & 2,3 & 2,2 & 2,0 & 1,9 \\
\hline & 2,0 & 1,9 & 1,8 & 1,7 & 1,8 & 1,7 & 1,5 & 1,7 & 1,5 \\
\hline \multirow[t]{4}{*}{1,50} & 1,0 & 2,5 & 3,8 & 2,7 & 2,4 & 1,8 & 3,0 & 2,1 & 1,8 \\
\hline & 1,3 & 2,3 & 2,5 & 2,1 & 2,0 & 1,8 & 2,1 & 1,7 & 1,6 \\
\hline & 1,5 & 2,1 & 2,1 & 1,8 & 1,8 & 1,8 & 1,8 & 1,6 & 1,5 \\
\hline & 2,0 & 1,7 & 1,6 & 1,5 & 1,6 & 1,4 & 1,4 & 1,4 & 1,4 \\
\hline
\end{tabular}

*gráfico de $\bar{X}$ com limites $\mu_{0} \pm 3.250 \sigma_{0} / \sqrt{4}$, e gráfico de $R$ com limite superior $5,257 \sigma_{0}$.

** gráfico de $\bar{X}$ com limites $\mu_{0} \pm 3.250 \sigma_{0} / \sqrt{5}$, e gráfico de $R$ com limite superior $5,433 \sigma_{0}$.

quando $\delta$ e/ou $\gamma$ aumenta. Para $\delta=0$ e $\gamma>0$, o NMA aumenta sempre que $d$ aumenta. Por exemplo, para $\mathrm{n}=5$; $\delta=0$ e $\gamma=1,5$, o NMA aumenta de 7,0 para 7,7 quando $d$ passa de 0 para 0,5 , e aumenta de 7,7 para 9,3 quando $d$ passa de 0,5 para 1,0 . Para $\delta>0$ e $\gamma=1,0$, o NMA diminui quando $d$ aumenta. Por exemplo, para $n=5 ; \delta=0,75 \mathrm{e}$ $\gamma=1,0, N M A$ diminui de 35,5 para 19,4 quando $d$ passa de 0 para 0,5 , e diminui de 19,4 para 14,6 quando $d$ passa de 0,5 para 1,0 .

\section{Gráfico de controle EWMA baseado na estatística de $\chi^{2}(\lambda)$}

O gráfico de controle da média móvel ponderada exponencialmente, mais conhecido como gráfico de EWMA, tem se mostrado útil na detecção de pequenas mudanças do processo (Reynolds e Stoumbos, 2001). Ampliando a possível utilização da estatística de Qui-quadrado não-central, no monitoramento de processos, propõe-se o gráfico de controle de EWMA baseado na seguinte estatística

$$
Y_{i}=r W_{i}+(1-r) Y_{i-1}, i=1,2, \ldots
$$

com $Y_{0}=E\left(W_{i} \mid \mu=\mu_{0}, \sigma=\sigma_{0}\right)$ e $0<r \leq 1$. O valor do parâmetro de amortecimento $r$ determina o peso da última observação $W_{i}$ na tomada de decisão quanto ao estado do processo, se sob controle ou não. Um valor maior de $r$ atribui maior peso à última observação, enquanto que um valor menor de $r$ atribui maior peso a $Y_{i-l}$, que é dependente de todas as observações, com exceção da última. O gráfico de EWMA sinaliza uma condição de falta de controle, sempre que $Y_{i}>k_{y} \sigma_{0}^{2}$, em que $k_{y}$ é o fator de abertura do limite superior de controle do gráfico. A Tabela 2 fornece para $n=5$, e diversos valores de $d, r, \delta$ e $\gamma$, o NMA para o gráfico de EWMA baseado na estatística de $\chi^{2}(\lambda)$ e para os gráficos conjuntos $\bar{X}$ e $R$. O fator de abertura $k_{y}$ é ajustado para que se tenha sempre um $N M A_{0}=433,0$ 
Tabela 2. Valores do NMA para os gráficos de $\bar{X}$ e $R$ e para o gráfico de $E W M A(n=5)$.

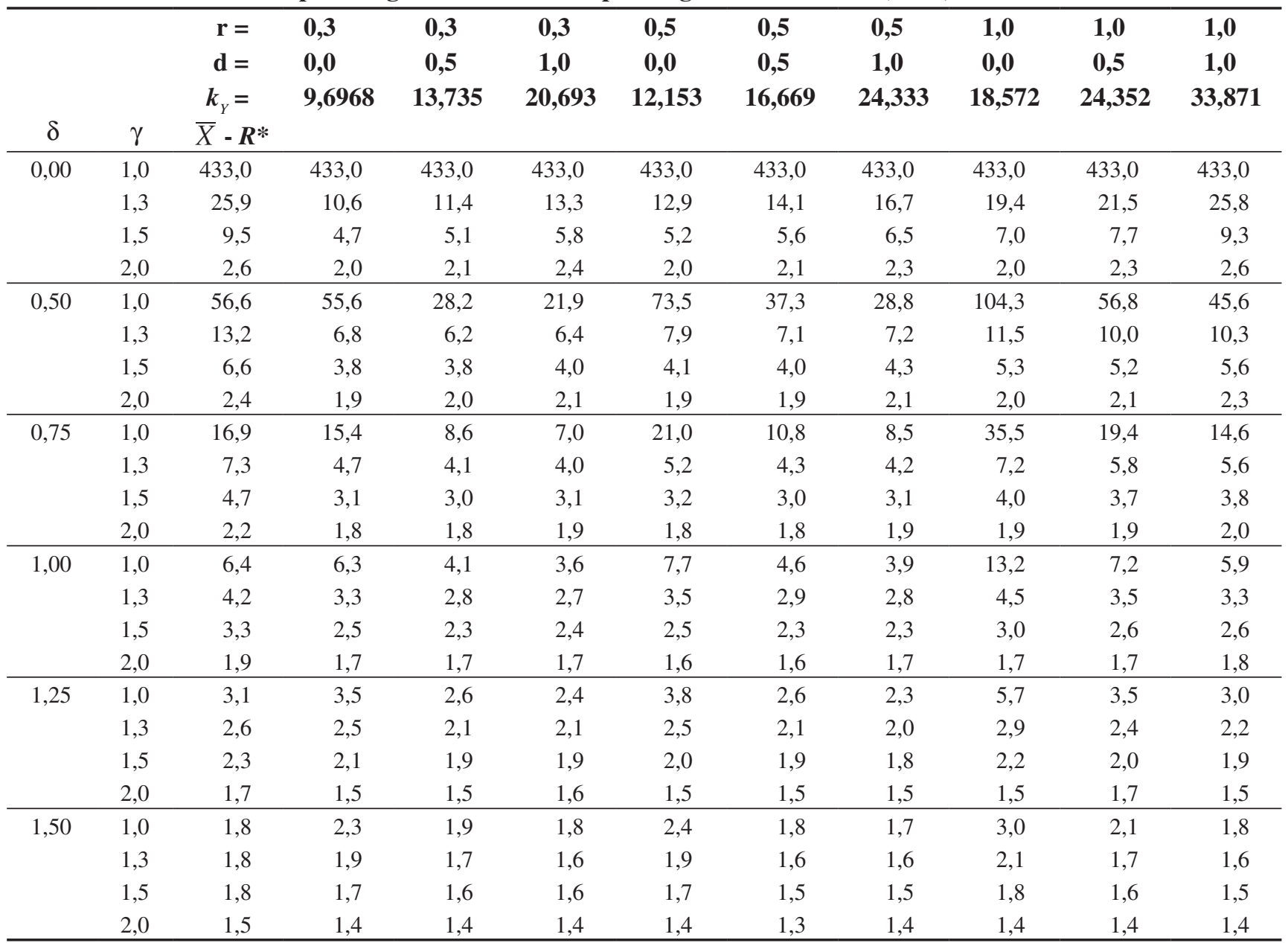

* gráfico de $\bar{X}$ com limites $\mu_{0} \pm 3.250 \sigma_{0} / \sqrt{5}$, e gráfico de $R$ com limite superior de controle $5,433 \sigma_{0}$.

Para o gráfico de $E W M A$, o número médio de amostras até o sinal dependerá do valor de $Y_{i}$ no instante em que os parâmetros do processo se alteram. Quando a mudança ocorre após um tempo de monitoramento relativamente longo, o NMA dependerá da distribuição estacionária de $Y_{i}$. Os valores do NMA da Tabela 2 foram obtidos a partir de uma cadeia Makov com 100 estados transientes, ou seja, a região do gráfico abaixo do limite superior de controle foi fragmentada em 100 partes iguais, ver Lucas e Saccucci (1990) para detalhes.

A análise da Tabela 2 leva às seguintes conclusões:

(1) O gráfico de EWMA com $d=0,5$ e 1,0 sempre detecta mudanças no processo com mais rapidez que os gráficos conjuntos de $\bar{X}$ e $R$, isto é, o $N M A$ do gráfico proposto é sempre menor que o NMA dos gráficos conjuntos. Por exemplo, para $\delta=0,5, \gamma=1,5$, e $n=5$, o NMA para os gráficos de $\bar{X}$ e $R$ é de 6,6 , enquanto que para o gráfico de $E W M A$ é de apenas $3,8(r=0,3$ e $d=0,5$ ).
(2) Para um dado $n, d$ e $r$, o NMA para o gráfico de $E W M A$ e para os gráficos conjuntos diminui sempre que $\delta$ e/ ou $\gamma$ aumentam.

(3) Para um dado $r$, valores maiores de $d$ são piores para a detecção de aumentos no desvio padrão $\sigma$, quando $\mu=\mu_{0}$. Por exemplo, para $r=0,3 ; \delta=0$ e $\gamma=1,3$, o $N M A$ aumenta de 10,6 para 11,4 quando $d$ passa de 0 para 0,5 , e de 11,4 para 13,3 quando $d$ passa de 0,5 para 1. Entretanto, para um dado $r$, valores maiores de $d$ são melhores para detecção de mudanças da média $\mu$, quando $\sigma=\sigma_{0}$. Por exemplo, para $r=0,3 ; \delta=0,75$ e $\gamma=1,0$, o NMA diminui de 15,4 para 8,6 quando $d$ passa de 0 para 0,5 , e de 8,6 para 7,0 quando $d$ passa de 0,5 para 1 .

(4) Para um dado $d$, valores maiores de $r$ são piores na detecção de deslocamentos da média $\mu$, quando $\sigma=\sigma_{0}$. Por exemplo, para $d=0,5, \delta=0,5$, e $\gamma=1,0, N M A$ aumenta de 28,2 para 37,3 quando $r$ passa de 0,3 para 0,5 , e de 37,3 para 56,8 , quando $r$ passa de 0,5 para 1,0 . 
(5) Para um dado $d$, valores maiores de $r$ são piores na detecção de aumentos na variabilidade, quando $\mu=\mu_{0}$. Por exemplo, para $d=0,5, \delta=0,0$, e $\gamma=1,3, N M A$ aumenta de 11,4 para 14,1 quando $r$ passa de 0,3 para 0,5 , e de 14,1 para 21,5 , quando $r$ passa de 0,5 para 1,0 .

A conclusão geral que se pode tirar das Tabelas 1 e 2 é que o esquema $E W M A$ proposto melhora a capacidade de detecção do gráfico de $\chi^{2}(\lambda)$.

A idéia de se utilizar um único gráfico de $E W M A$, no monitoramento da média e da variância do processo, foi inicialmente considerada por Domangue e Patch (1991). O gráfico por eles proposto é baseado na seguinte estatística

$$
A_{i}=r\left|Z_{i}\right|^{\beta}+(1-r) A_{i-1}
$$

para $i=1,2, \ldots, 0<r \leq 1, \beta=0,5$ ou 2,0 , e

$$
Z_{i}=\sqrt{n}\left(\bar{x}_{i}-\mu_{0}\right) / \sigma_{0},
$$

em que $\mu_{0}$ e $\sigma_{0}^{2}$ são os valores da média e da variância do processo quando este está em controle, $n$ é o tamanho das amostras, e $\bar{X}_{i}$ é a média da i-ésima amostra, respectivamente.

Para $n=1$, e $d=0$, o gráfico de EWMA se reduz a um dos gráficos de Domangue e Patch (1991), em que $\beta=2,0$. De fato, para $W_{i}=\left(X_{i 1}-\mu_{0}\right)^{2}$, os valores do $N M A$ coincidem com aqueles apresentados por Domangue e Patch (1991).

\section{Exemplo}

O gráfico de EWMA proposto neste artigo foi planejado para substituir os gráficos de controle de $\bar{X}$ e $R$, em uso no monitoramento de um sistema de enchimento de garrafas. A quantidade de líquido $X$, em cada garrafa, é a característica de qualidade de interesse. Ocasionalmente, certas impurezas do líquido entopem parcialmente a tubulação por onde flui o líquido. Esta causa especial altera a média e aumenta a variabilidade do processo. Para eliminar a causa especial é necessário limpar a tubulação. Limpezas desnecessárias devem ser evitadas, pois demandam paradas do processo.

O monitoramento do processo é feito por meio da retirada, a cada 20 minutos, de uma amostra de cinco garrafas $(n=5$ e $h=20)$. Os valores das médias amostrais são plotados no gráfico de $\bar{X}$ com limites de controle dados por $\mu_{0} \pm 3,250 \sigma_{0} / \sqrt{n}$, e os valores das amplitudes amostrais são plotados no gráfico de $R$ com limite superior dado por $5,433 \sigma_{0}$.

A Tabela 3 fornece os valores dos volumes de 75 garrafas (isto é, 15 amostras de tamanho 5). Os valores de $W_{i}$ e $Y_{i}$, para cada uma das quinze amostras, estão nas últimas duas colunas. $\mathrm{O}$ volume especificado de cada garrafa é de 600,00 mililitros (ml), ou seja, o valor alvo da média é 600,00 $\mathrm{ml}$. Quando isento de impurezas, o desvio padrão do processo é igual a $2,00 \mathrm{ml}$. Foram adotados para os seguintes valores: $d=0,5 ; r=0,3$ e $Y_{0}=29,18$. A título de ilustração, a Figura 1 apresenta o gráfico proposto de EWMA com os 15 pontos amostrais (valores de $Y_{i}$ da Tabela 3).

Os seguintes pontos são favoráveis ao uso do gráfico de EWMA em substituição aos gráficos conjuntos de $\bar{X}$ e $R$ :

(a) A simplicidade operacional: é sempre mais fácil operar um único gráfico de controle do que dois gráficos, especialmente quando muitas características de qualidade são controladas ao mesmo tempo.

Tabela 3. Valores de $X_{i j}, W_{i}$ e $Y_{i}$.

\begin{tabular}{cccccccc}
\hline $\begin{array}{c}\text { \# da amostra } \\
(i)\end{array}$ & $\boldsymbol{X}_{\boldsymbol{i} 1}$ & $\boldsymbol{X}_{\boldsymbol{i} 2}$ & $\boldsymbol{X}_{i 3}$ & $\boldsymbol{X}_{\boldsymbol{i} 4}$ & $\boldsymbol{X}_{i 5}$ & $\boldsymbol{W}_{\boldsymbol{i}}$ & $\boldsymbol{Y}_{\boldsymbol{i}}$ \\
\hline 1 & 599,83 & 598,00 & 600,93 & 601,60 & 600,25 & 13,78 & 24,56 \\
2 & 598,30 & 599,34 & 598,80 & 599,92 & 596,49 & 36,44 & 28,12 \\
3 & 600,00 & 598,22 & 597,11 & 600,63 & 600,89 & 24,08 & 26,91 \\
4 & 603,22 & 602,02 & 600,14 & 598,30 & 603,53 & 49,25 & 33,61 \\
5 & 596,77 & 596,56 & 600,15 & 599,70 & 602,23 & 41,52 & 35,98 \\
6 & 601,47 & 596,73 & 598,01 & 601,12 & 601,03 & 27,40 & 33,41 \\
7 & 599,98 & 598,98 & 599,16 & 597,73 & 601,64 & 19,62 & 29,27 \\
8 & 597,26 & 600,67 & 599,35 & 600,54 & 598,59 & 22,88 & 27,35 \\
9 & 601,05 & 600,61 & 599,18 & 597,69 & 598,72 & 19,61 & 25,03 \\
10 & 602,41 & 602,90 & 600,00 & 602,29 & 599,78 & 39,24 & 29,29 \\
11 & 597,76 & 601,11 & 596,47 & 601,08 & 601,06 & 31,05 & 29,82 \\
12 & 599,51 & 601,02 & 601,22 & 597,49 & 599,52 & 16,81 & 25,92 \\
14 & 597,95 & 600,93 & 597,95 & 604,39 & 604,29 & 62,92 & 37,02 \\
\hline
\end{tabular}




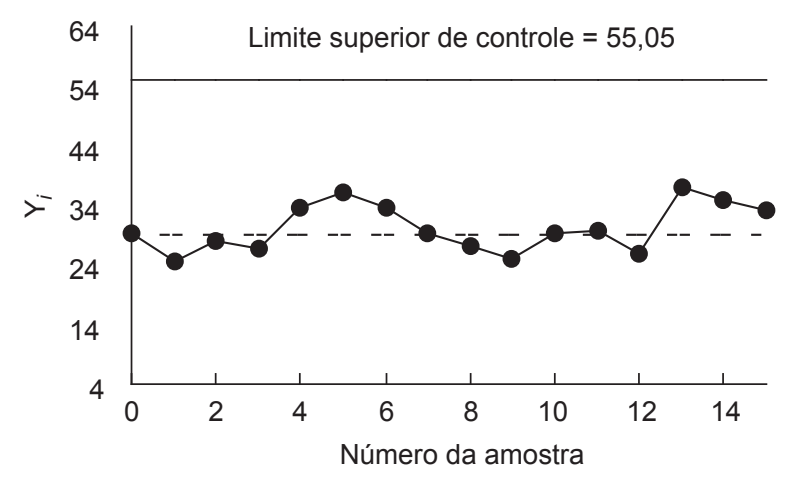

Figura 1. Gráfico de $E W M A$ com 15 valores da estatística $Y_{i}\left(r=0,3 ; d=0,5\right.$, e $\left.Y_{0}=29,18\right)$.

(b) A sensibilidade: quando comparado com os gráficos conjuntos de $\bar{X}$ e $R$, o gráfico de EWMA detecta mudanças no processo com mais rapidez. Por exemplo, os gráficos conjuntos demandam, em média, 7,3 amostras de tamanho cinco para detectar um deslocamento moderado da média do processo $(\delta=0,75)$, acompanhado de um pequeno aumento da variabilidade $(\gamma=1,30)$. Com o gráfico proposto $(r=0,3$ e $d=0,5)$, este número se reduz a 4,1, vide Tabela 2 .

\section{Conclusões}

Neste trabalho, mostrou-se que é possível projetar um único gráfico de controle para o monitoramento simultâneo da média e da variabilidade do processo. O gráfico proposto não é apenas mais eficiente do que os gráficos conjuntos de $\bar{X}$ e $R$, no sentido de que ele detecta com mais rapidez alterações da média do processo, acompanhada ou não de aumentos da variabilidade, mas também ele é mais simples em termos operacionais, pois a cada instante de amostragem, o usuário lida com o cálculo e a plotagem de valores de uma única estatística. Quando é importante detectar pequenas mudanças no processo, $\mathrm{o}$ usuário deve considerar o gráfico de controle de EWMA. O esquema $E W M A$ melhora em muito a capacidade de detecção do gráfico de controle de $\chi^{2}(\lambda)$, em especial de pequenas perturbações.

\section{Agradecimentos}

Graças a uma revisão cuidadosa dos revisores várias imperfeições foram eliminadas, somos profundamente gratos. O suporte financeiro para a realização deste trabalho foi fornecido pelo Conselho Nacional de Desenvolvimento Científico e Tecnológico (CNPq).

\section{Referências Bibliográficas}

ALBIN, S. L.; KANG, L.; SHEHA, G. An $X$ and EWMA chart for individual observations. Journal of Quality Technology, Milwaukee, v. 29, n. 1, p. 41-48, jan./mar. 1997.

CHEN, G.; CHENG, S. W.; XIE, H. Monitoring process mean and variability with one EWMA chart. Journal of Quality Technology, Milwaukee, v. 33, n. 2, p. 223-233, abr./jun. 2001.

COSTA, A. F. B. Joint economic design of $\bar{X}$ and R control charts for processes subject to two independent assignable causes. IIE Transactions, Norcross, v. 25, n. 1, p. 27-33, jan. 1993.

Joint $\bar{X}$ and $\mathrm{R}$ charts with variable parameters. IIE Transactions, Norcross, v. 30, n. 6, p. 505-514, jun. 1998.

Joint $\bar{X}$ and $\mathrm{R}$ charts with variable sample sizes and sampling intervals. Journal of Quality Technology, Milwaukee, v. 31, n. 4, p. 387-397, out./dez. 1999.

COSTA, A. F. B.; RAHIM, M. A. Economic design of $\bar{X}$ and $\mathrm{R}$ charts under Weibull shock models. Quality and Reliability Engineering International, New York, v. 16, n.2, p. 143-156, mar./abr. 2000.
Economic design of charts with variable parameters: the Markov chain approach. Journal of Applied Statistics, Park, v. 28, n. 7, p. 875-885, set. 2001.

Joint $\bar{X}$ and $\mathrm{R}$ charts with two stage samplings.

Quality and Reliability Engineering International, New York, v. 20, n. 7, p. 699-708, nov./dez. 2004.

COSTA, A. F. B.; EPPRECHTE. K.; CARPINETTI, L. C. R. (2004) Controle Estatístico de Qualidade, 2. ed. São Paulo: Atlas, 2005. 334 p.

DOMANGUE, R.; PATCH, S. C. Some omnibus exponentially weighted moving average statistical process monitoring schemes. Technometrics, Milwaukee, v. 33, n. 3, p. 299-313, ago./out. 1991.

GAN, F. F. Joint monitoring of process mean and variance using exponentially weighted moving average control charts. Technometrics, Milwaukee, v. 37, n. 4, p. 446-453, nov./jan. 1995.

LUCAS, J. M.; SACCUCCI, M. S. Exponentially weighted moving average control schemes: properties and enhancements. Technometrics, Milwaukee, v. 32, n. 1, p. 1-12, fev./abr. 1990. 
RAHIM, M. A.; COSTA, A. F. B. Joint economic design of $\bar{X}$ and $\mathrm{R}$ charts under Weibull shock models. International Journal of Production Research. Loughborough, v. 28, n. 13, p. 2871-2889, dez. 2000.

REYNOLDS, M. R.; STOUMBOS, Z.G. Monitoring the process mean and variance using individual observations and variable sampling intervals. Journal of Quality Te- chnology, Milwaukee, v. 33, n. 2, p. 181-205, abr./jun. 2001.

SHAMMA, S. E.; AMIN, R. W. An EWMA quality control procedure for jointly monitoring the mean and the variance, International Journal of Quality \& Reliability Management, Toller Lane, v. 10, n. 7, p. 58-67, dez. 1993.

\title{
THE NONCENTRAL CHI SQUARE STATISTIC APPLIED TO PROCESS CONTROL
}

\begin{abstract}
It is standard practice to use joint charts in process control, one designed to detect shifts in the mean and the other to detect changes in the variance of the process. In this paper, we propose the use of a single chart to control both mean and variance. Based on the noncentral chi square statistic, the single chart is faster in detecting shifts in the mean and increases in variance than its competitor, the joint $\bar{X}$ and $R$ charts. The noncentral chi square statistic can also be used with the EWMA procedure, particularly in the detection of small mean shifts, accompanied or not by slight increases in variance.
\end{abstract}

Keywords: noncentral chi square chart, joint $\bar{X}$ and $R$ charts, average run length. 